\title{
Hepatic inflammation precedes steatosis and is mediated by visceral fat accumulation
}

\author{
Breno Picin Casagrande, Daniel Vitor de Souza, Daniel Araki Ribeiro, Alessandra Medeiros, Luciana Pellegrini Pisani \\ and Debora Estadella
}

Biosciences Department, Institute of Health and Society, Federal University of São Paulo, Santos, São Paulo, Brazil

Correspondence should be addressed to D Estadella: estadella.debora@gmail.com

\begin{abstract}
The negative aspects of unhealthy eating on obesity and hepatic health are well described. The axis between the adipose tissue and the liver participates in most of the damage caused to this tissue regarding obesogenic diets (OD). At the same time that the effects of consuming simple carbohydrates and saturated fatty acids are known, the effects of the cessation of its intake are scarce. Withdrawing from OD is thought to improve health; despite some studies had shown improvement in hepatic conditions in the long-term, short-term studies were not found. Therefore, we aimed to determine how OD intake and withdrawal would influence visceral and hepatic fat accumulation and inflammation. To this end, male 60-days-old Wistar rats received standard chow $(n=16)$ or a high-sugar/ high-fat diet (HSHF) for 30 days $(n=32)$, a cohort of the HSHF-fed animals was then kept $48 \mathrm{~h}$ on standard chow $(n=16)$. In opposition to the generally reported, the results indicate that hepatic inflammation preceded hepatic steatosis. Additionally, inflammatory markers on the liver positively correlated visceral adipokines and visceral fat accumulation mediated them in a deposit-dependent manner. At the same time, a 48-h withdrawal was capable of reverting most of the risen inflammatory mediators, although MyD88 and TNF $\alpha$ persisted and serum non-HDL cholesterol was higher than control levels.
\end{abstract}

Key Words

- hepatic inflammation

- hepatic steatosis

- visceral adipose tissue

- obesogenic diet

- withdrawal

\section{Introduction}

The negative aspects of obesogenic diets (OD) on adipose tissue accumulation, hepatic fat accumulation (steatosis), and inflammation are well described (Van Herck et al. 2017, Bortolin et al. 2018, Casagrande et al. 2019). Obesity and non-alcoholic fatty liver disease (NAFLD) are two parts of the same role, respectively, as causation and consequence of the metabolic syndrome (Kanwar \& Kowdley 2016). It is believed that following the adipocytehepatocyte axis, an increase in adipose tissue deposits drives an increase in the hepatic fat content, mainly as triacylglycerol (TAG) (Smith \& George 2009). NAFLD can progress to non-alcoholic steatohepatitis (NASH), with higher inflammation (Kanwar \& Kowdley 2016). https://joe.bioscientifica.com https://doi.org/10.1530/JOE-20-0073
(C) 2020 Society for Endocrinology Published by Bioscientifica Ltd.
Printed in Great Britain
The adipocyte-hepatocyte axis can promote an overactivation of the toll-like receptors pathway and the production of inflammatory cytokines. Likewise, simple carbohydrates and fatty acids from the diet produce this effect (Wagnerberger et al. 2012, Rocha et al. 2016, Totsch et al. 2017). This pathway can be mediated by myeloid differentiation factor 88 (MyD88) and lead to the phosphorylation and nuclear translocation of the nuclear factor kappa B (NFkB) culminating in cytokine production (Lu et al. 2008).

The harms caused by unhealthy eating are widely known (Morris et al. 2015). Meanwhile, despite improving eating habits being recommended for populations 
consuming ODs (Jensen et al. 2014), few studies describe the effects of stopping consuming an OD. In the longterm, stopping OD intake, that is, withdrawing from it, was reported to improve hepatic health (Das et al. 2017). At the same time, it decreased liver steatosis, but not pro-inflammatory macrophage activation (Zamarron et al. 2017). Conversely, we were not able to find studies applying a short-term withdrawal (WTD) and evaluating those parameters in the liver.

Considering that the harms associated with unhealthy eating patterns are proposed to decrease after dietary improvement and that some consequences may persist at short- and long-term withdrawal (Hazarika et al. 2016, Liu et al. 2016a, Das et al. 2017, Zamarron et al. 2017), our objective was to determine how visceral and hepatic fat contents and hepatic inflammation would respond to OD intake and a short-term withdrawal and, if so, whether they would be related or not.

\section{Materials and methods}

\section{Ethics}

The present study was approved by the ethics committee of the 'Federal University of Sao Paulo' ( $n^{\circ} 4641210318$ ). All procedures were carried out under the ethical principles to animal research, according to the 'National Board of Control for Animal Experimentation,' following national and international guidelines.

\section{Experimental protocol}

Thirty 60-day-old male young adult Wistar rats (Rattus norvegicus), 210-260 g, habituated to the bioterium for 7 days were randomly assigned into three groups. Animals were held in cages $(40 \times 35 \times 15 \mathrm{~cm})$ of $3-4$ individuals during the experiment, in a $12 \mathrm{~h}$ light: $12 \mathrm{~h}$ darkness cycle, at $22 \pm 2^{\circ} \mathrm{C}$, receiving water and chow, the standard or an obesogenic diet (high-sugar and high-fat diet, HSHF) ad libitum. The first group (Control group, Ct, $n=10$ ) received the standard chow for 30 days; the second (HSHF-fed, Hd, $n=10)$ received the modified diet for 30 days; and the third (HSHF-fed + withdrawal, Hw, $n=10$ ) received the modified diet for 30 days and the standard chow for 2 days (Fig. 1). The standard chow used was Nuvilab CR (Quimtia, Colombo, Brazil). The modified diet (Table 1) consisted of grounded standard chow added with sweetened condensed milk (Nestle) and lard (Sadia, São Paulo, Brazil), to characterise an HSHF. In order to ensure adequate nutrition, it was added of casein (Labsynth, Diadema, Brazil), soybean oil (Bunge, Gaspar, Brazil), vitamins (RHOSTER, Araçoiaba da Serra, Brazil), and minerals (RHOSTER). At the end of the protocol, all animals were anaesthetised with isoflurane and killed. The ARRIVE checklist was used in the preparation of this manuscript (Kilkenny et al. 2010).

An additional cohort (Ct, $n=6$; $\mathrm{Hd}, n=6$; $\mathrm{Hw}, n=6$ ) was carried out with the same experimental design but was ked by perfusion with $0.9 \%$ sodium chloride in water (Labsynth) and subsequently with $4 \%$ paraformaldehyde in PB (phosphate buffer, $0.1 \mathrm{M}, \mathrm{pH}$ 7.4) (fixing solution) (Labsynth). The livers were kept in fixing solution for 48 $\mathrm{h}$ and transferred to a $70 \%$ alcohol solution.

\section{Serum lipids}

Total serum cholesterol (\#76, Labtest, Lagoa Santa, Brazil), serum high-density lipoprotein cholesterol (HDL) (\#13, Labtest), and serum triacylglycerol (\#87, Labtest) were determined by an enzymatic method using a commercial colourimetric kit following manufacturer's guidelines. Non-HDL cholesterol was calculated subtracting HDL from total cholesterol.

\section{Hepatic lipids}

The content of hepatic triacylglycerol was determined by an adaptation of the method of total lipids extraction of Folch et al. (1957). A solution of chloroform-methanolwater (2:1:0.5) (Labsynth) was added to each sample, which was homogenised and centrifuged at $400 \boldsymbol{g}$ for $10 \mathrm{~min}$, twice. The precipitate was evaporated and resuspended with Triton-X 3\% (Labsynth). The triacylglycerol (\#87,

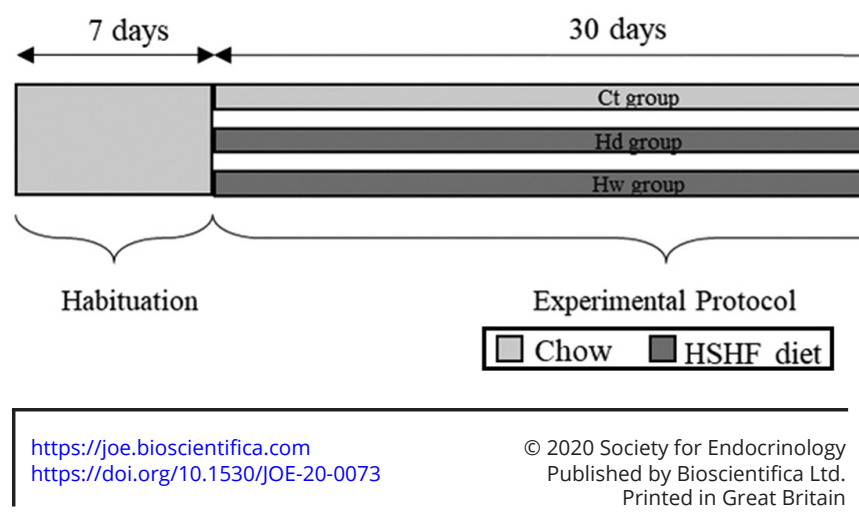

Figure 1

Experimental design. Duration of habituation and experimental protocol and chow provided during those phases. Ct, Control group; Hd, HSHF-fed; Hw, HSHF-fed + 48 h withdrawal; HSHF, high-sugar and high-fat diet. https://joe.bioscientifica.com https://doi.org/10.1530/JOE-20-0073 
Table 1 Composition and nutritional information of the diets.

\begin{tabular}{|c|c|c|}
\hline & Standard chow & HSHF \\
\hline \multicolumn{3}{|l|}{ Components (g/1000 g) } \\
\hline Nuvilab CR1® & 1000 & 334.96 \\
\hline Sweetened condensed milk & - & 400 \\
\hline Lard & - & 86.57 \\
\hline Casein & - & 36.07 \\
\hline Sucrose & - & 113.37 \\
\hline Soybean oil & - & 12.63 \\
\hline Mineral mix & - & 11.72 \\
\hline Vitamin mix & - & 3.68 \\
\hline \multicolumn{3}{|l|}{ Nutrients (g/1000 g) } \\
\hline Carbohydrates & 600 & 511 \\
\hline Sucrose & 0 & 33 \\
\hline Fibre & 70 & 23 \\
\hline Protein & 220 & 150 \\
\hline Lipids & 40 & 132 \\
\hline Saturated fatty acids & 0 & 55 \\
\hline \multicolumn{3}{|l|}{ Energy (\%) } \\
\hline Carbohydrates & 63 & 53 \\
\hline Sucrose & 0 & 35 \\
\hline Protein & 26 & 16 \\
\hline Lipids & 11 & 31 \\
\hline Saturated fatty acids & 0 & 19 \\
\hline Energy (kcal/100 g) & 3340 & 3833 \\
\hline
\end{tabular}

Labtest) and cholesterol (\#76, Labtest) content were determined by an enzymatic method using a commercial colourimetric kit. The percentage of hepatic cholesterol was calculated, dividing its value by the sum of hepatic cholesterol and triacylglycerol (hepatic cholesterol/ (hepatic cholesterol + hepatic triacylglycerol)).

\section{Protein content analysis}

Tissue samples were individually homogenised in lysis buffer (100 mM Tris-HCl, $10 \mathrm{mM}$ sodium pyrophosphate, 10 $\mathrm{mM}$ sodium orthovanadate, $2 \mathrm{mM}$ phenylmethylsulfonyl fluoride, $0.04 \%$ bovine lung aprotinin, Sigma-Aldrich; $10 \mathrm{mM}$ EDTA, $100 \mathrm{mM}$ sodium fluoride, 1\% Triton, Labsynth) and centrifuged at 20,000 $\boldsymbol{g}$ for $40 \mathrm{~min}$ at $4^{\circ} \mathrm{C}$. The supernatant was collected, and the protein content was assessed with Bradford reagent. Interleukin 6, 10, 1 $\beta$, and TNF $\alpha$ protein contents were determined by the ELISA method (R\&D Systems) following the manufacturer's instructions.

Western blotting, as previously described (Santamarina et al. 2018), was carried out to determine the protein content of phosphorylated nuclear factor kappa B p65 (pNFкBp65, p-p65) (1:1200, \#3033 Cell Signaling Technology), myeloid differentiation factor 88 (MyD88) (1:5000, \#4283S Cell Signaling Technology), and $\beta$-actin (1:5000, \#3700 Cell Signaling Technology), used as loading control. The respective secondary antibodies followed the primary antibodies, anti-mouse (1:20,000, \#7076, Cell Signaling Technology) or anti-rabbit IgG, HRP-linked (1:20,000, \#7074, Cell Signaling Technology). Images were obtained using chemiluminescence intensifier scanner UVITEC (Cambridge, UK), and quantification was performed with Scion Image software Beta 4.0.2 (NIH).

\section{Hepatic histology}

Posterior to fixation, the livers were dehydrated gradually in ethanol, diaphanized in xylol, and incorporated in paraffin. The paraffin blocks were cut in $3-\mu \mathrm{m}$ sections and stained with haematoxylin-eosin to histopathological evaluation. The criteria followed the proposed by Aguiar et al. (2011).

\section{Statistical analysis}

Sample size calculation was performed on $G^{\star}$ Power v3.1.9.4, the a priori test accounted for one-way ANOVA, considering the effect size $\mathrm{f}$ as $0.6, \alpha$ as 0.05 , and power as 0.8 ; resulting in 30 individuals. Data was compiled with Microsoft Excel for Office 365 (16.0.12026.20312) and analysed with JASP v0.11.1. The data were assessed for normality and homoscedasticity, outliers were determined by ROUT test. For data with parametric sample distribution, we used one-way ANOVA and Tukey's post hoc in order to verify differences among and between groups, respectively; for data with nonparametric sample distribution, we used Kruskal-Wallis test and Dunn's post hoc. To determine effect size among groups, ANOVA's sum of squares was used in order to calculate Cohen's $f$, while for between groups, means, S.D., and sample size were used to determine Hedges' $g$, which is a small-sample-size correction for Cohen's $d$. Depending on the normality of data's distribution, Pearson's or Spearman's approach was used to determining correlations; the correlated data were then applied in mediation models and subsequently in structural equation modeling. For all analyses, the statistically significant limit was set at $P<0.05$.

\section{Results}

High-sugar and high-fat diet intake and withdrawal

After consuming the HSHF diet, the Hd group presented greater body mass, serum TAG, mesenteric, epidydimal and 
retroperitoneal fat deposits, hepatic protein expression of p-p65 (Fig. 2A), Myd88 (Fig. 2B), IL6, IL10, IL1ß, and TNFa (Fig. 3 and Tables 2, 3). Withdrawing from HSHF promoted lower serum TAG, mesenteric fat accumulation, and protein content of hepatic p-p65 (Fig. 2A), IL6, IL10, IL1 $\beta$ (Fig. 3), and partially lower body mass, and hepatic TNF $\alpha$ (Fig. 3). WTD resulted in higher serum cholesterol and serum non-HDL cholesterol, as well as the percentage of hepatic cholesterol. Still, neither HSHF intake nor WTD changed hepatic triacylglycerol content nor hepatic cholesterol content (Tables 2 and 3).

In the histopathological analysis, we observed no signs of steatosis nor focal inflammation under microscopical evaluation. All animals ( $n=18, \mathrm{Ct}=6, \mathrm{Hd}=6, \mathrm{Hw}=6)$ rated ' 0 ' considering the criteria adopted (Aguiar et al. 2011).

(Fig. 4A, B and C).
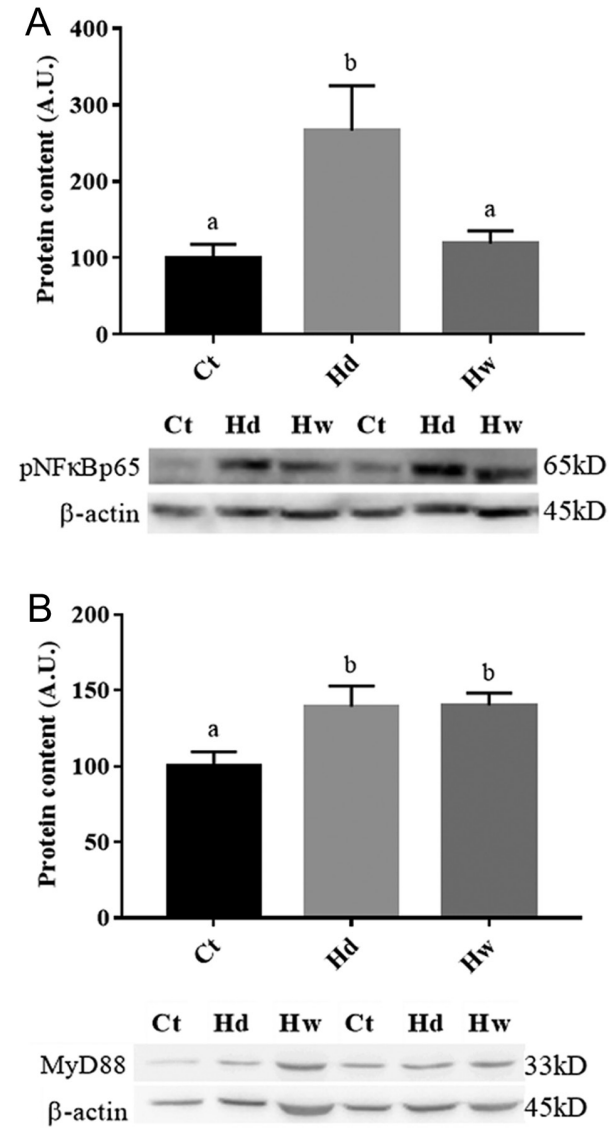

\section{Figure 2}

Graphical representation of hepatic protein levels assessed by Western blotting. (A) Hepatic protein content of phosphorylated nuclear factor kappa B p65 (pNFkBp65); (B) myeloid differentiation factor 88 (MyD88); (A.U.) Arbitrary units; values are presented as mean \pm S.E.M. and are expressed relative to the $\mathrm{Ct}$ group mean. Ct, control group; $\mathrm{Hd}$, HSHF-fed group; Hw, HSHF-fed $+48 \mathrm{~h}$ withdrawal group; Different letters indicate $P<0.05$ between groups. The number of animals per group is described in Table 2.
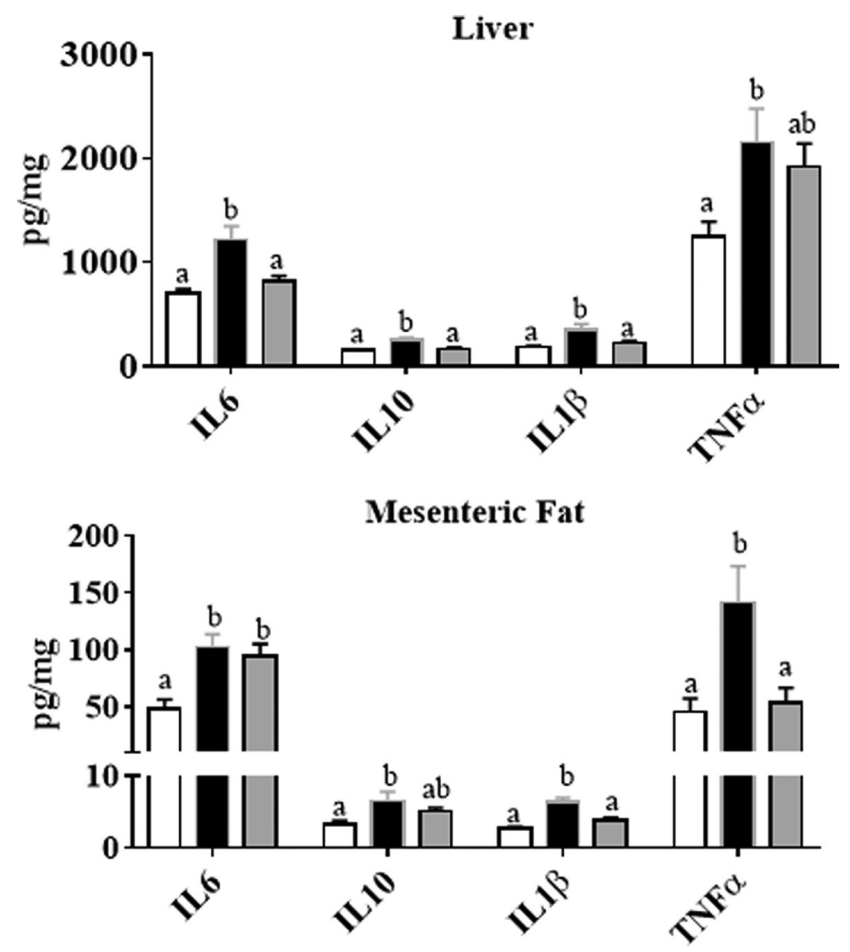

Epididymal Fat

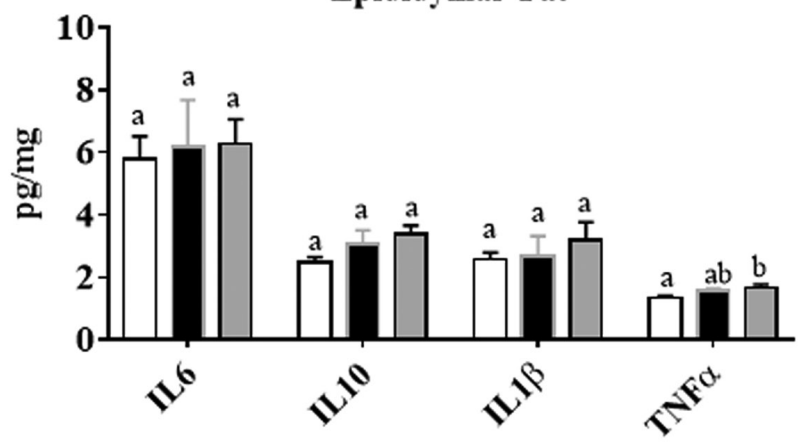

Retroperitoneal Fat

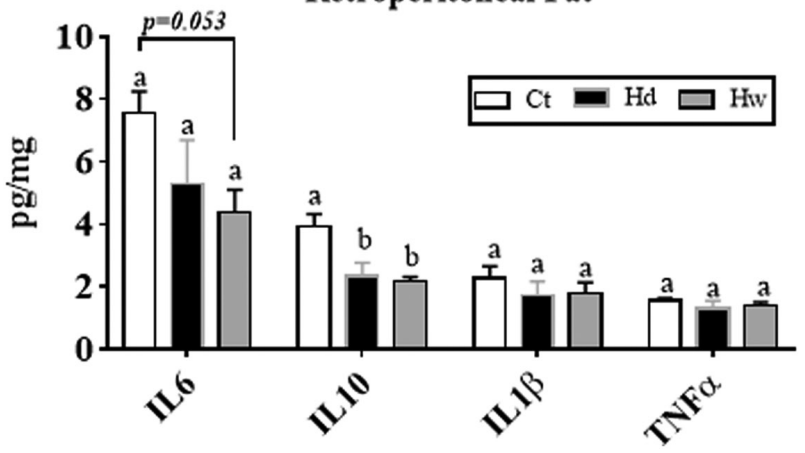

Figure 3

Graphical representation of cytokine concentrations assessed by ELISA on the liver, mesenteric fat, epidydimal fat, and retroperitoneal fat. Values are presented as mean \pm S.E.M. in picograms per milligram of protein. $\mathrm{Ct}$, control group; Hd, HSHF-fed group; Hw, HSHF-fed +48 h withdrawal group; Different letters indicate $P<0.05$ between groups. The number of animals per group is described in Table 2. 
Table 2 Summary of results.

\begin{tabular}{l}
\hline Parameters \\
Body mass \\
Serum cholesterol (mg/dL) \\
Serum HDLc (mg/dL) \\
Serum n-HDLc (mg/dL) \\
Serum triacylglycerol (mg/dL) \\
Hepatic cholesterol (g/100 g) \\
Hepatic triacylglycerol (g/100 g) \\
Hepatic cholesterol (\% of lipids) \\
Mesenteric fat (g/100 g BM) \\
Epididymal fat (g/100 g BM) \\
Retroperitoneal fat (g/100 g BM) \\
Hepatic pNFkBp65 (A.U.) \\
Hepatic MyD88 (A.U.) \\
Liver \\
IL6 (pg/mg) \\
IL10 (pg/mg) \\
IL1 $\beta$ (pg/mg) \\
TNF $\alpha$ (pg/mg) \\
Mesenteric fat \\
IL6 (pg/mg) \\
IL10 (pg/mg) \\
IL1 $\beta$ (pg/mg) \\
TNF $\alpha$ (pg/mg) \\
Epididymal fat \\
IL6 (pg/mg) \\
IL10 (pg/mg) \\
IL1 $\beta$ (pg/mg) \\
TNF $\alpha$ (pg/mg) \\
Retroperitoneal fat \\
IL6 (pg/mg) \\
IL10 (pg/mg) \\
IL1 $\beta$ (pg/mg) \\
TNF $\alpha$ (pg/mg) \\
\hline
\end{tabular}

\begin{tabular}{c}
\hline Ct group \\
\hline Mean \pm S.E.M. $(n)$ \\
\hline $398.10 \pm 6.98(10)^{\mathrm{a}}$ \\
$107.10 \pm 8.70(10)^{\mathrm{a}}$ \\
$39.09 \pm 2.85(9)^{\mathrm{a}}$ \\
$64.39 \pm 6.66(9)^{\mathrm{a}}$ \\
$108.00 \pm 6.80(10)^{\mathrm{a}}$ \\
$20.64 \pm 1.37(10)^{\mathrm{a}}$ \\
$1.99 \pm 0.19(10)^{\mathrm{a}}$ \\
$51.57 \pm 1.97(10)^{\mathrm{a}}$ \\
$4.61 \pm 0.42(10)^{\mathrm{a}}$ \\
$7.57 \pm 0.86(10)^{\mathrm{a}}$ \\
$7.25 \pm 0.74(10)^{\mathrm{a}}$ \\
$100.00 \pm 17.99(6)^{\mathrm{a}}$ \\
$100.00 \pm 9.36(9)^{\mathrm{a}}$ \\
$704.60 \pm 36.15(10)^{\mathrm{a}}$ \\
$152.60 \pm 11.12(10)^{\mathrm{a}}$ \\
$185.10 \pm 11.51(10)^{\mathrm{a}}$ \\
$1245.00 \pm 145.40(7)^{\mathrm{a}}$
\end{tabular}

$$
\begin{array}{r}
47.73 \pm 8.38(8)^{a} \\
3.92 \pm 0.45(8)^{a} \\
2.65 \pm 0.26(7)^{a} \\
45.63 \pm 11.70(8)^{a}
\end{array}
$$

$$
\begin{gathered}
5.8 \pm 0.72(9)^{a} \\
2.47 \pm 0.18(9)^{a} \\
2.58 \pm 0.22(10)^{a} \\
1.34 \pm 0.08(10)^{a} \\
7.56 \pm 0.68(9)^{a} \\
3.94 \pm 0.39(9)^{a} \\
2.26 \pm 0.40(9)^{a} \\
1.57 \pm 0.07(9)^{a}
\end{gathered}
$$

\begin{tabular}{c}
\hline Hd group \\
\hline Mean \pm S.E.M. $(n)$ \\
\hline $436.20 \pm 11.93(9)^{\mathrm{b}}$ \\
$125.00 \pm 10.36(7)^{\mathrm{ab}}$ \\
$34.77 \pm 2.63(7)^{\mathrm{a}}$ \\
$90.20 \pm 8.84(7)^{\mathrm{ab}}$ \\
$161.70 \pm 13.67(7)^{\mathrm{b}}$ \\
$20.15 \pm 0.99(7)^{\mathrm{a}}$ \\
$1.67 \pm 0.18(7)^{\mathrm{a}}$ \\
$55.39 \pm 2.65(7)^{\mathrm{a}}$ \\
$8.37 \pm 0.56(9)^{\mathrm{b}}$ \\
$17.77 \pm 1.81(9)^{\mathrm{b}}$ \\
$16.05 \pm 1.21(9)^{\mathrm{b}}$ \\
$266.70 \pm 58.39(6)^{\mathrm{b}}$ \\
$139.20 \pm 13.65(7)^{\mathrm{b}}$ \\
$1219.00 \pm 129.80(7)^{\mathrm{b}}$ \\
$255.20 \pm 19.02(7)^{\mathrm{b}}$ \\
$353.90 \pm 51.43(7)^{\mathrm{b}}$ \\
$2157.00 \pm 321.70(6)^{\mathrm{b}}$
\end{tabular}

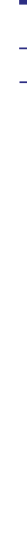

\begin{tabular}{ccrr} 
Hw group & & $\begin{array}{c}\text { ANOVA } \\
\text { Pevalue }\end{array}$ \\
\cline { 1 - 1 } $426.80 \pm 9.62(10)^{\mathrm{ab}}$ & & 0.024 \\
$141.80 \pm 9.93(10)^{\mathrm{b}}$ & & 0.044 \\
$44.48 \pm 2.93(10)^{\mathrm{a}}$ & & 0.082 \\
$97.29 \pm 9.18(10)^{\mathrm{b}}$ & & 0.021 \\
$101.7 \pm 1.92(10)^{\mathrm{a}}$ & & $<0.001$ \\
$23.66 \pm 0.83(10)^{\mathrm{a}}$ & & 0.073 \\
$1.66 \pm 0.11(10)^{\mathrm{a}}$ & & 0.257 \\
$59.10 \pm 1.43(10)^{\mathrm{b}}$ & & 0.029 \\
$6.30 \pm 0.54(8)^{\mathrm{a}}$ & & $<0.001$ \\
$16.86 \pm 1.92(10)^{\mathrm{b}}$ & & $<0.001$ \\
$14.62 \pm 0.92(10)^{\mathrm{b}}$ & & $<0.001$ \\
$119.30 \pm 16.06(6)^{\mathrm{a}}$ & & 0.011 \\
$140.00 \pm 8.16(10)^{\mathrm{b}}$ & & 0.013 \\
& & \\
$815.50 \pm 54.92(10)^{\mathrm{a}}$ & & $<0.001$ \\
$169.50 \pm 11.75(10)^{\mathrm{a}}$ & & $<0.001$ \\
$223.10 \pm 22.38(10)^{\mathrm{a}}$ & & 0.001 \\
$2023.00 \pm 227.20(8)^{\mathrm{ab}}$ & & 0.029
\end{tabular}

$102.30 \pm 11.08(6)^{b}$

$6.53 \pm 1.26(7)^{\mathrm{b}}$

$6.27 \pm 0.68(6)^{b}$

$141.30 \pm 31.95(6)^{b}$

$6.19 \pm 1.49(7)^{\mathrm{a}}$
$3.07 \pm 0.43(7)^{\mathrm{a}}$
$2.67 \pm 0.65(7)^{\mathrm{a}}$
$1.55 \pm 0.09(5)^{\mathrm{ab}}$
$5.3 \pm 1.39(7)^{\mathrm{a}}$
$2.35 \pm 0.41(7)^{\mathrm{b}}$
$1.73 \pm 0.44(5)^{\mathrm{a}}$
$1.3 \pm 0.25(7)^{\mathrm{a}}$

$\begin{array}{rr}94.28 \pm 10.75(8)^{\mathrm{b}} & 0.002 \\ 4.99 \pm 0.54(7)^{\mathrm{ab}} & 0.031 \\ 3.84 \pm 0.35(6)^{\mathrm{a}} & <0.001 \\ 53.23 \pm 13.42(6)^{\mathrm{a}} & 0.006\end{array}$

$6.28 \pm 0.79(9)^{a} \quad 0.931$

$3.19 \pm 0.58(10)^{\mathrm{a}} \quad 0.622$

$1.66 \pm 0.11(9)^{\mathrm{b}} \quad 0.047$

$4.38 \pm 0.73(8)^{\mathrm{a}} \quad 0.056$

$2.16 \pm 0.15(8)^{\mathrm{b}} \quad 0.002$

$\begin{array}{ll}1.79 \pm 0.34(6)^{\mathrm{a}} & 0.579\end{array}$

$1.41 \pm 0.10(8)^{a} \quad 0.437$
$3.38 \pm 0.28(9)^{a} \quad 0.088$

Different letters indicate $P<0.05$ between groups, for Tukey's post hoc and for one-way ANOVA.

A.U., arbitrary units; BM, body mass; Ct, Control group; Hd, HSHF-fed; Hw, HSHF-fed + withdrawal; IL6, interleukin 6; IL10, interleukin 10; IL1 $\beta$, interleukin 1 beta; MyD88, myeloid differentiation factor 88; pNFkBp65, phosphorylated nuclear factor kappa B p65; TNFo, tumor necrosis factor alfa.

\section{Serum lipids and hepatic inflammation}

Serum lipids positively correlated with hepatic inflammation markers. Total serum cholesterol $\left(r=0.45, r^{2}=0.20, P=0.04\right)$ and non-HDL serum cholesterol $\left(r=0.53, r^{2}=0.28, P=0.02\right)$ positively correlated with hepatic TNF $\alpha$. Serum TAG positively correlated with hepatic IL10 $\left(r=0.48, r^{2}=0.23\right.$, $P=0.01)$. The percentage of hepatic cholesterol positively correlated with epidydimal $\left(r=0.43, r^{2}=0.18, P=0.02\right)$ and retroperitoneal fat $\left(r=0.48, r^{2}=0.23, P=0.01\right)$ and with hepatic TNF $\alpha\left(r=0.49, r^{2}=0.24, P=0.02\right)$.

\section{Visceral fat accumulation and hepatic inflammation}

Visceral fat accumulation deposit-dependently positively correlated with hepatic inflammation. The mesenteric fat (g/100 g) was positively correlated with hepatic protein content of p-p65, MyD88, IL6, IL10, and IL1 $\beta$; the epidydimal (g/100 g), with hepatic MyD88, IL10, IL1 $\beta$, and TNF $\alpha$; and the retroperitoneal (g/100 g), with hepatic MyD88, IL6, IL10, IL1 $\beta$, and TNF $\alpha$ (Table 4).

\section{Visceral adipose tissue inflammation and hepatic inflammation}

Considering the role played by visceral adipose tissue (VAT) in hepatic inflammation, we performed cytokine analysis in mesenteric, epididymal, and retroperitoneal fat tissues. HSHF intake caused mesenteric fat IL6, IL10, IL1 $\beta$, and TNF $\alpha$ to be higher and retroperitoneal fat IL10 to be lower than those of Ct group (Tables 2 and 3). After withdrawal, IL6 was kept elevated, IL10 was similar to both Ct's and Hd's, and IL1 $\beta$ and TNF $\alpha$ returned to control-like 
Table 3 Effect size among and between groups.

\begin{tabular}{l}
\hline Parameters \\
\hline Body mass \\
Serum cholesterol (mg/dL) \\
Serum HDL (mg/dL) \\
Serum non-HDL (mg/dL) \\
Serum triacylglycerol (mg/dL) \\
Hepatic cholesterol (g/100 g) \\
Hepatic cholesterol (\% of lipids) \\
Hepatic triacylglycerol (g/100 g) \\
Mesenteric fat (g/100 g BM) \\
Epididymal fat (g/100g BM) \\
Retroperitoneal fat (g/100 g BM) \\
Hepatic NFkB (A.U.) \\
Hepatic MyD88 (A.U.) \\
Liver \\
IL6 (pg/mg) \\
IL10 (pg/mg) \\
IL1 $\beta$ (pg/mg) \\
TNF $\alpha$ (pg/mg) \\
Mesenteric fat \\
IL6 (pg/mg) \\
IL10 (pg/mg) \\
IL1 $\beta$ (pg/mg) \\
TNF $\alpha$ (pg/mg) \\
Epididymal fat \\
IL6 (pg/mg) \\
IL10 (pg/mg) \\
IL1 $\beta$ (pg/mg) \\
TNF $\alpha(\mathrm{pg} / \mathrm{mg})$ \\
Retroperitoneal fat \\
IL6 (pg/mg) \\
IL10 (pg/mg) \\
IL1 $\beta$ (pg/mg) \\
TNF $\alpha(\mathrm{pg} / \mathrm{mg})$ \\
\hline
\end{tabular}

\begin{tabular}{c}
\hline Ct vs Hd Hedges' $\boldsymbol{g}$ \\
\hline-1.24 \\
-1.81 \\
1.48 \\
-3.32 \\
-5.03 \\
0.38 \\
-0.55 \\
0.55 \\
-2.31 \\
-2.31 \\
-2.78 \\
-1.60 \\
-1.71 \\
-2.08 \\
-2.32 \\
-1.77 \\
-1.41 \\
\\
-2.03 \\
-1.24 \\
-2.73 \\
-1.59 \\
\\
0.74 \\
1.33 \\
0.44 \\
0.55 \\
0.74 \\
1.33 \\
0.44 \\
0.55 \\
\end{tabular}

\begin{tabular}{c}
\hline Ct vs Hw Hedges' $\mathbf{g}$ \\
\hline-1.03 \\
-3.56 \\
-1.79 \\
-3.93 \\
1.20 \\
-2.55 \\
-1.32 \\
0.65 \\
-1.22 \\
-1.89 \\
-2.67 \\
-0.90 \\
-1.42 \\
-0.72 \\
-0.45 \\
-0.65 \\
-1.36 \\
-1.62 \\
-1.18 \\
-1.45 \\
-0.22 \\
1.47 \\
1.88 \\
0.42 \\
0.60 \\
1.47 \\
1.88 \\
0.42 \\
0.60 \\
\end{tabular}

\begin{tabular}{ccc}
\hline Hd vs Hw Hedges' $\boldsymbol{g}$ & & Among groups Cohen's $\boldsymbol{f}$ \\
\cline { 1 - 1 } \cline { 1 - 1 } 0.27 & 0.58 \\
-1.58 & 0.55 \\
-3.27 & 0.49 \\
-0.76 & 0.63 \\
6.49 & 1.17 \\
-3.69 & 0.49 \\
-0.60 & 0.58 \\
0.02 & 0.35 \\
0.80 & 1.13 \\
0.15 & 0.99 \\
0.42 & 1.37 \\
1.22 & 0.91 \\
-0.03 & 0.68
\end{tabular}

Number of rats per group is described in Table 2.

A.U., arbitrary units; BM, body mass; Ct, Control group; Hd, HSHF-fed; Hw, HSHF-fed + withdrawal; IL6, interleukin 6; IL10, interleukin 10; IL1 $\beta$, interleukin 1 beta; MyD88, myeloid differentiation factor 88; pNFkBp65, phosphorylated nuclear factor kappa B p65; TNF $\alpha$, tumor necrosis factor alfa.

levels in mesenteric fat; IL10 continued lower compared to Ct's; TNFo in epididymal fat was higher than Ct's.

Moreover, VAT cytokines correlated with most of the hepatic inflammatory parameters presented. Mesenteric fat IL6 positive correlated with hepatic IL1 $\beta$ and TNFo; IL10 with hepatic IL6, IL1 $\beta$, and TNF $\alpha$; IL1 $\beta$ with hepatic MyD88, IL6, 1 $\beta$, and TNF $\alpha$; and TNF $\alpha$ with hepatic MyD88 and IL1 $\beta$ (Table 5). Epididymal fat IL1 $\beta$ positively correlated with hepatic MyD88 $\left(r=0.46, r^{2}=0.21, P=0.022\right)$ and its TNF $\alpha$ positively correlated with hepatic TNF $\alpha(r=0.49$, $\left.r^{2}=0.24, P=0.029\right)$. Retroperitoneal fat IL10 negatively correlated with hepatic IL6 $\left(r=-0.48, r^{2}=0.23, P=0.019\right)$.

\section{Hepatic inflammation is mediated by visceral fat accumulation}

To pursue a further relationship, we used mediation and structural equation modeling. Mediation models

\begin{tabular}{lr}
\hline https://joe.bioscientifica.com & (C) 2020 Society for Endocrinology \\
https://doi.org/10.1530/JOE-20-0073 & Published by Bioscientifica Ltd. \\
Printed in Great Britain
\end{tabular}

can be seen as sets of linear regressions and show that the total effect observed from the intervention can be broken down into components (e.g. direct effect and indirect effect) displaying the contribution of the proposed mediator on the final result (Montoya \& Hayes 2017). Structural equation modeling is a confirmatory factor analysis that allows questions involving multiple regression analyses to be answered, setting relationships between several variables of interest at the same time (Ullman \& Bentler 2017).

Following the correlations found, we tested mediation models and structural equation modeling. Serum lipids and hepatic cholesterol (\% of hepatic lipids) showed no mediation of intake's nor withdrawal's effect. On the structural equation modeling, we were able to see that mesenteric fat accumulation mediated the effect of HSHF intake on IL1 $\beta$ (Table 6 (1)) and p-p65 (Table 6 ' 2 '), whereas p-p65 mediated the effect of mesenteric fat on 

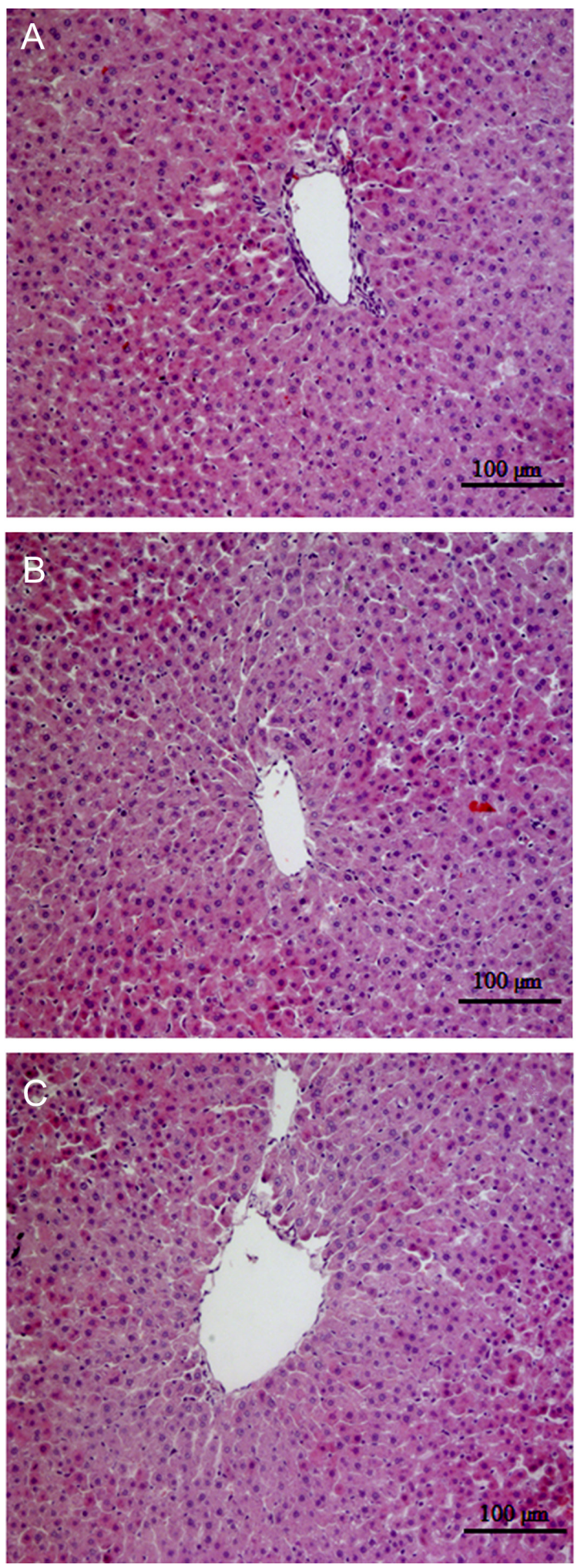

Figure 4

Hepatic tissue microphotographs (20x) of haematoxylin-eosin staining. (A) Control group; (B) Hd, HSHF-fed group; (C) Hw, HSHF-fed + $48 \mathrm{~h}$ withdrawal group. ( $n=6$ per group). A full color version of this figure is available at https://doi.org/10.1530/JOE-19-0553.
IL1 $\beta$ (Table 6 (3)). Therefore, the effect followed the equation 'HSHF $\rightarrow$ Mesenteric Fat $\rightarrow$ pNFkBp65 $\rightarrow$ IL1 $\beta^{\prime}$ (Table 6 (A)). Hence, the increment in IL1 $\beta$ following HSHF intake was determined by mesenteric fat accumulation and higher hepatic p-p65.

In the same way, IL6 mediated both HSHF intake and withdrawal effect on IL1 $\beta$ (Table 6 (41) and (51)), the HSHF intake effect on IL10 (Table 6 (42)), and partially the withdrawal effect on IL10 (Table 6 (52)), whereas, IL10 mediated IL6 effect on IL1 $\beta$ (Table 6 (6)). Therefore, the effect followed the equation 'HSHF/WTD $\rightarrow$ IL6 $\rightarrow$ IL10 $\rightarrow$ IL1 $\beta$ ' (Table 6 (B)). Hence, the increment in IL10 and IL1 $\beta$ was determined or partially determined by higher hepatic IL6 (Fig. 5).

Additionally, in simple mediation models, epidydimal fat accumulation mediated the effect of HSHF intake on MyD88 (Fig. 6A and Table 7 (1) and (2)). Also, the effect of HSHF intake on TNF $\alpha$ was mediated by retroperitoneal fat accumulation (Fig. 6B and Table 7 (3) and (4)). Hence, epidydimal fat and retroperitoneal fat accumulation, respectively, determined higher hepatic MyD88 and TNF $\alpha$.

\section{Discussion}

The consumption of an obesogenic diet is known to increase body mass, VAT, and to promote hepatic inflammation and hepatic steatosis (Lozano et al. 2016, Casagrande et al. 2019). Still, there is not much information regarding these parameters when terminating the intake of OD. Our results indicate that a 48-h dietary improvement was efficient in returning most of the analysed parameters to control-like levels. Still, body fat accumulation, which decreases more slowly over time, persisted (Martire et al. 2014, Rinnankoski-Tuikka et al. 2014).

On the other hand, total serum cholesterol, mainly non-HDL cholesterol, composed majorly by LDL and VLDL, was higher. This rise could indicate higher lipid export in response to WTD, supported by a higher percentage of hepatic cholesterol. Interestingly, serum lipid levels were not correlated with intra-hepatic lipids nor VAT accumulation. As there was no similarity between fat accumulation and serum cholesterol, the observed greater hepatic lipid exportation might be the decisive factor.

In a broader perspective, higher non-HDL cholesterol is strongly linked with long-term risk for cardiovascular diseases (Brunner et al. 2019). This rise is of concern, pondering the frequent cycling of diet and weight in the population with obesity. Body mass fluctuations affecting 
Table 4 Correlations between visceral fat accumulation and hepatic inflammation.

\begin{tabular}{|c|c|c|c|}
\hline \multirow[b]{2}{*}{ Hepatic parameters } & \multicolumn{3}{|c|}{ Mesenteric fat } \\
\hline & $r$ & $r^{2}$ & $P$ value \\
\hline pNFкBp65 & 0.678 & 0.460 & 0.002 \\
\hline MyD88 & 0.426 & 0.181 & 0.038 \\
\hline IL6 & 0.446 & 0.199 & 0.025 \\
\hline IL10 & 0.557 & 0.310 & 0.004 \\
\hline IL1 $\beta$ & 0.616 & 0.379 & 0.001 \\
\hline $\operatorname{TNF} \alpha$ & 0.400 & 0.160 & 0.081 \\
\hline
\end{tabular}

\begin{tabular}{|c|c|c|}
\hline \multicolumn{3}{|c|}{ Epidydimal fat } \\
\hline$r$ & $r^{2}$ & $P$ value \\
\hline 0.158 & 0.025 & 0.507 \\
\hline 0.647 & 0.419 & $<0.001$ \\
\hline 0.363 & 0.132 & 0.063 \\
\hline 0.460 & 0.212 & 0.016 \\
\hline 0.446 & 0.199 & 0.020 \\
\hline 0.595 & 0.354 & 0.004 \\
\hline
\end{tabular}

\begin{tabular}{|c|c|c|}
\hline \multicolumn{3}{|c|}{ Retroperitoneal fat } \\
\hline$r$ & $r^{2}$ & $P$ value \\
\hline 0.408 & 0.166 & 0.074 \\
\hline 0.477 & 0.228 & 0.014 \\
\hline 0.523 & 0.274 & 0.005 \\
\hline 0.419 & 0.176 & 0.030 \\
\hline 0.511 & 0.261 & 0.006 \\
\hline 0.675 & 0.456 & 0.001 \\
\hline
\end{tabular}

IL6, interleukin 6; IL10, interleukin 10; IL1ß, interleukin 1 beta; MyD88, myeloid differentiation factor 88; pNFkBp65, phosphorylated nuclear factor kappa B p65; TNF $\alpha$, tumor necrosis factor alfa.

cardiovascular health in the population with obesity are long known (Jeffery et al. 1992, Zeigler et al. 2018, Zou et al. 2019).

There is a relationship between VAT accumulation and hepatic tissue metabolism (Smith \& George 2009, Barchetta et al. 2019, Cornide-Petronio et al. 2019, Franchitto et al. 2019, Ishtiaq et al. 2019). Adipocytehepatocyte lipid accumulation axis is proposed to be one of the most critical participants in NAFLD development (Smith \& George 2009). Interestingly, adipose tissue inflammation, but not only fat accumulation, was linked to NAFLD (Asrih \& Jornayvaz 2013). Our results lead us to believe that VAT inflammation links with hepatic inflammation and precedes NAFLD.

Hepatic inflammation occurred despite hepatic TAG accumulation following HSHF intake and is related to visceral fat accumulation and inflammation. We have recently shown that chronic OD intake, 13 weeks of a high-fat and high-fructose diet, can drive VAT accumulation and hepatic inflammation despite no significant hepatic TAG increase (Casagrande et al. 2019).

Combined fat and sugar seem to induce earlier inflammation and later TAG accumulation. Intrahepatic TAG accumulation was seen after 1 and 5 weeks of highfat feeding (Shang et al. 2017, Santamarina et al. 2019); though, HSHF feeding increased hepatic TAG after 12 weeks, but not after 1 nor 4 weeks (Chiba et al. 2016).
Interestingly, free fatty acids (FFA) accumulation, but not TAG, was associated with NAFLD development (Liu et al. 2016b). Liu et al. (2016b) found that accumulation of TAG happened with 8 weeks of high-fat diet intake, whereas accumulation of FFA only after 16 weeks. Additionally, plasmatic FFA was suggested as an indicator for predicting advanced fibrotic states on NAFLD patients (Zhang et al. 2014). Concomitantly, time-dependent accumulation of FFA may reflect the progression of NAFLD (Konstantynowicz-Nowicka et al. 2019), and therefore, our protocol's length was perhaps not sufficient to induce those changes and thus NAFLD.

Fat tissue releases several factors - hormones, nutrients, cytokines - in the bloodstream, some of them are linked to hepatic inflammatory status (e.g. adiponectin, free fatty acids, and TNF $\alpha$ ), which may have contributed to the results observed here (Musso et al. 2005, Asrih \& Jornayvaz 2013, Berk \& Verna 2016, Ishtiaq et al. 2019). Conversely, VAT cytokines positively correlated with hepatic inflammatory markers. Indeed, visceral fat accumulation and chronic low-grade inflammation play a leading role in the development and progression of metabolic syndrome following unhealthy diets (Zafar et al. 2018).

Interleukin 6 and TNF $\alpha$ are non-exclusive acute-phase cytokines (Feghali \& Wright 1997). If their rise in the liver were solely dependant on the external stimuli (e.g. HSHF

Table 5 Correlations between mesenteric fat and hepatic inflammation.

\begin{tabular}{|c|c|c|c|c|c|c|c|c|c|c|c|c|}
\hline \multirow{3}{*}{$\begin{array}{l}\text { Hepatic } \\
\text { parameters }\end{array}$} & \multicolumn{12}{|c|}{ Mesenteric fat } \\
\hline & \multicolumn{3}{|c|}{ IL6 } & \multicolumn{3}{|c|}{ IL10 } & \multicolumn{3}{|c|}{ IL1 $\beta$} & \multicolumn{3}{|c|}{ TNF $\alpha$} \\
\hline & $r$ & $r^{2}$ & $P$ value & $r$ & $r^{2}$ & $P$ value & $r$ & $r^{2}$ & $P$ value & $r$ & $r^{2}$ & $P$ value \\
\hline pNFкBp65 & 0.172 & 0.030 & 0.575 & 0.130 & 0.017 & 0.660 & 0.337 & 0.114 & 0.284 & 0.342 & 0.117 & 0.276 \\
\hline MyD88 & 0.340 & 0.116 & 0.143 & 0.262 & 0.069 & 0.264 & 0.503 & 0.253 & 0.040 & 0.513 & 0.264 & 0.029 \\
\hline IL6 & 0.420 & 0.176 & 0.051 & 0.431 & 0.186 & 0.045 & 0.498 & 0.248 & 0.018 & 0.456 & 0.208 & 0.066 \\
\hline IL10 & 0.375 & 0.141 & 0.086 & 0.322 & 0.104 & 0.143 & 0.405 & 0.164 & 0.063 & 0.475 & 0.226 & 0.056 \\
\hline IL1 $\beta$ & 0.637 & 0.406 & 0.003 & 0.790 & 0.624 & $<0.001$ & 0.638 & 0.407 & 0.003 & 0.597 & 0.356 & 0.024 \\
\hline TNF $\alpha$ & 0.497 & 0.247 & 0.026 & 0.732 & 0.536 & $<0.001$ & 0.635 & 0.403 & 0.003 & 0.463 & 0.214 & 0.082 \\
\hline
\end{tabular}

IL6, interleukin 6; IL10, interleukin 10; IL1 $\beta$, interleukin 1 beta; MyD88, myeloid differentiation factor 88; pNFkBp65, phosphorylated nuclear factor kappa B p65; $r$, Pearson's correlation coefficient; TNF $\alpha$, tumour necrosis factor alfa; $\rho$, Spearman's correlation coefficient. 
Table 6 Structural equation modeling.

\begin{tabular}{l}
\hline \\
\hline (A) HSHF $\rightarrow$ Mesenteric Fat $\rightarrow$ pNFkBp65 $\rightarrow$ IL1 \\
(1) HSHF $\rightarrow$ Mesenteric Fat $\rightarrow$ IL1 $\beta$ \\
$(2) \mathrm{HSHF} \rightarrow$ Mesenteric Fat $\rightarrow$ pNFkBp65 \\
$(3)$ Mesenteric Fat $\rightarrow$ pNFkBp65 $\rightarrow$ IL1 $\beta$ \\
(B) HSHF/WTD $\rightarrow$ IL6 $\rightarrow$ IL10 $\rightarrow$ IL1 $\beta$ \\
$(41)$ HSHF $\rightarrow$ IL6 $\rightarrow$ IL1 $\beta$ \\
(42) HSHF $\rightarrow$ IL6 $\rightarrow$ IL10 \\
(51) WTD $\rightarrow$ IL6 $\rightarrow$ IL1 $\beta$ \\
(52) WTD $\rightarrow$ IL6 $\rightarrow$ IL10 \\
(6) IL6 $\rightarrow$ IL10 $\rightarrow$ IL1 $\beta$
\end{tabular}

\begin{tabular}{|c|c|}
\hline $\mathbf{a} \boldsymbol{\beta}(\mathrm{z})$ & $\mathbf{b} \boldsymbol{\beta}(\mathrm{z})$ \\
\hline $0.62(3.21)^{a}$ & $\begin{array}{l}0.60(2.79)^{a} \\
0.54(2.50)^{a}\end{array}$ \\
\hline $0.60(2.79)^{a}$ & $0.69(3.96)^{a}$ \\
\hline $0.87(4.75)^{a}$ & $\begin{array}{l}0.54(2.90)^{a} \\
0.73(6.47)^{a}\end{array}$ \\
\hline $\begin{array}{c}-0.65 \\
(-3.53)^{a}\end{array}$ & $\begin{array}{l}0.54(2.90)^{a} \\
0.73(6.47)^{a}\end{array}$ \\
\hline $0.73(6.47)^{\mathrm{a}}$ & $0.69(2.36)^{a}$ \\
\hline
\end{tabular}

\begin{tabular}{c}
\hline \multicolumn{1}{c}{$\mathbf{c}^{\prime} \boldsymbol{\beta}(\mathrm{z})$} \\
\hline $0.16(0.74)$ \\
$0.25(1.16)$ \\
$0.12(0.66)$ \\
$0.30(1.28)$ \\
$0.27(1.88)$ \\
$-0.24(-1.13)$ \\
$-0.29(-2.26)^{\mathrm{a}}$ \\
$0.03(0.13)$ \\
\hline
\end{tabular}

\begin{tabular}{c}
$\begin{array}{c}\text { Mediated effect } \\
(\mathrm{a} \times \mathrm{b}) \boldsymbol{\beta}(\mathrm{z})\end{array}$ \\
\hline \\
$0.37(2.10)^{\mathrm{a}}$ \\
$0.33(1.97)^{\mathrm{a}}$ \\
$0.41(2.28)^{\mathrm{a}}$ \\
\\
$0.47(2.48)^{\mathrm{a}}$ \\
$0.64(3.83)^{\mathrm{a}}$ \\
$-0.35(-2.24)^{\mathrm{a}}$ \\
$-0.47(-3.10)^{\mathrm{a}}$ \\
$0.50(2.22)^{\mathrm{a}}$ \\
\hline
\end{tabular}

\begin{tabular}{c}
\hline $\begin{array}{c}\text { Total effect c } \\
\left(a \times b+c^{\prime}\right) \boldsymbol{\beta}(z)\end{array}$ \\
\hline $0.53(2.57)^{a}$ \\
$0.58(2.93)^{a}$ \\
$0.54(2.50)^{a}$ \\
$0.77(3.87)^{a}$ \\
$0.91(5.32)^{a}$ \\
$-0.59(-2.94)^{a}$ \\
$-0.76(-4.47)^{a}$ \\
$0.54(2.90)^{a}$
\end{tabular}

Effect size (standardised $\beta$ ), z value, and significance of the models' coefficients. a indicate $P<0.05$.

$\rightarrow$, orientation of effect; $a$, effect of predictor $(X)$ on mediator $(M) ; b$, effect of mediator $(M)$ on outcome $(Y)$ controlling for predictor $(X)$; $C^{\prime}$, direct effect of predictor $(\mathrm{X})$ on outcome $(\mathrm{Y})$; HSHF, high-sugar and high fat diet; IL6, interleukin 6; IL10, interleukin 10; IL1 $\beta$, interleukin 1 beta; MyD88, myeloid differentiation factor 88; pNFkBp65, phosphorylated nuclear factor kappa B p65; WTD, withdrawal.

diet), IL6 and TNF $\alpha$ levels would be both lower after the $48 \mathrm{~h}$ withdrawal, according to their peak concentration and half-life post-challenge (Sharma et al. 1992, de Vos et al. 1994). The reduction of IL6 and subsequently of IL10 and IL1 $\beta$ and the maintenance of TNF $\alpha$ levels may indicate a persisting stimulus other than HSHF intake. The determinant could be retroperitoneal fat accumulation, as proposed by the mediation model (Fig. 6B), or epididymal fat TNF $\alpha$ and mesenteric fat IL6, both positively correlated with hepatic TNF $\alpha$.

Based on the data from structural equation modeling, it is possible to propose an inflammatory cascade playing an essential role in the mechanisms of HSHF intake and VAT effect on hepatic inflammation. The production of IL1 $\beta$ following p-p65 (Fig. 5) can be a consequence of the activation of 'nucleotide-binding oligomerisation domain and leucine-rich repeat-containing receptor' 3 (NLRP3) (Liu et al. 2017), whereas IL1 $\beta$ production following IL10 increase might indicate an interplay of the mammalian target of rapamycin complex 1 and NLRP3 (mTORCNLRP3) pathway (Kabat \& Pearce 2017).

In this sense, IL1 $\beta$ would be one of the triggers and one of the results of the pathways' activation, since it promotes the cleavage of pro-IL1 $\beta$ into its active form via NLRP3-caspase1 (Liu et al. 2017, Jin \& Fu 2019). Associated with hypoxia, lipopolysaccharides (LPS), and $\mathrm{TNF} \alpha$ increase in the adipose tissue, the NLRP3-IL1 $\beta$ pathway plays an essential role in inflammation and obesity-associated comorbidities (Unamuno et al. 2019). Remarkably, mesenteric fat cytokines, consequently TNF $\alpha$, were positively correlated with hepatic IL1 $\beta$, reinforcing this relationship.

Until the discovery of adipose tissue regulatory activity in energy metabolism, participating in the control of cerebral and peripheral mechanisms, it was acknowledged as an inert organ (Zhang etal. 1994, Kershaw $\&$ Flier 2004). Among the main effectors produced are leptin, adiponectin, adipokines, and regulatory peptides

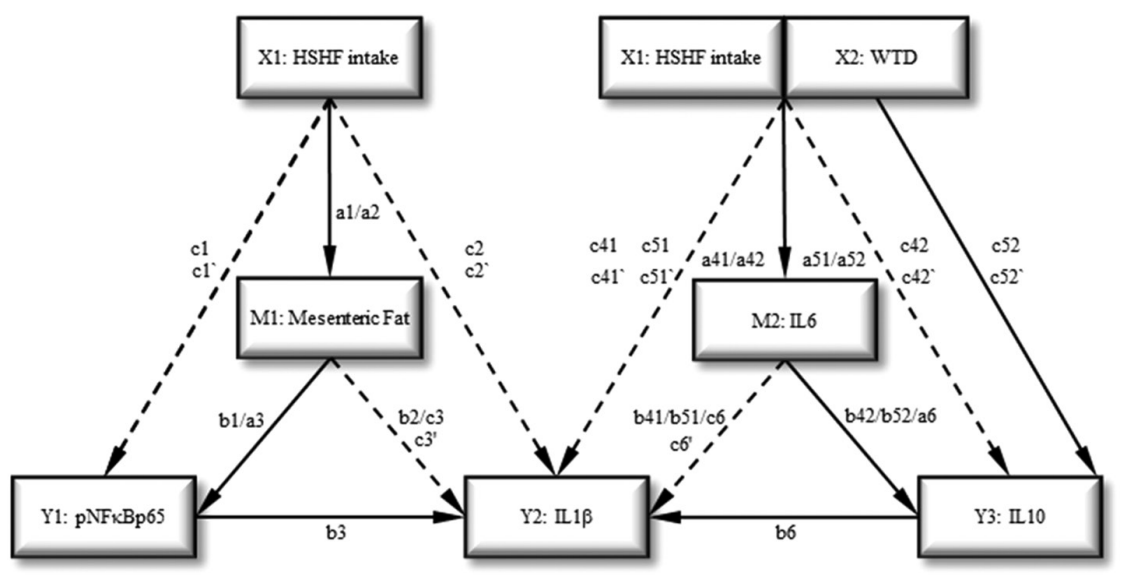

\section{Figure 5}

Structural equation modeling for HSHF intake effect on hepatic pNFKBp65, II1 $\beta$, and IL10. (1) and (2) display the models for mesenteric fat mediation of the intake effect on pNFkBp65 and IL1 $\beta$; (3) displays the effect of mesenteric fat on IL1 $\beta$ mediated by pNFkBp65; (41) and (51) display the models for IL6 mediation of intake effect on IL10 and IL1 $\beta$; while (42) and (52) display the models for IL6 mediation of WTD on IL10 and IL1 $\beta$; (6) displays the effect of IL6 on IL1 $\beta$ mediated by IL10. (a) Effect of the predictor $(X)$ on the mediator (M); (b) effect of the mediator (M) on the outcome $(Y)$ controlling for the predictor $(X)$; $\left(C^{\prime}\right)$ direct effect of the predictor $(X)$ on the outcome (Y); (continuous arrow) significant effect; (dashed arrow) non-significant effect. 


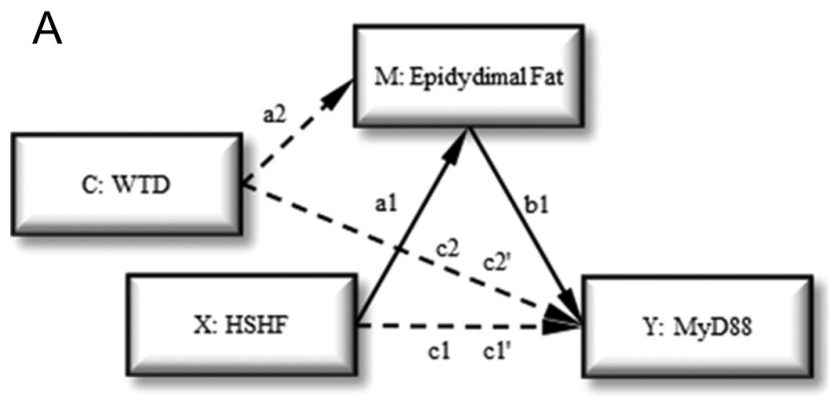

B

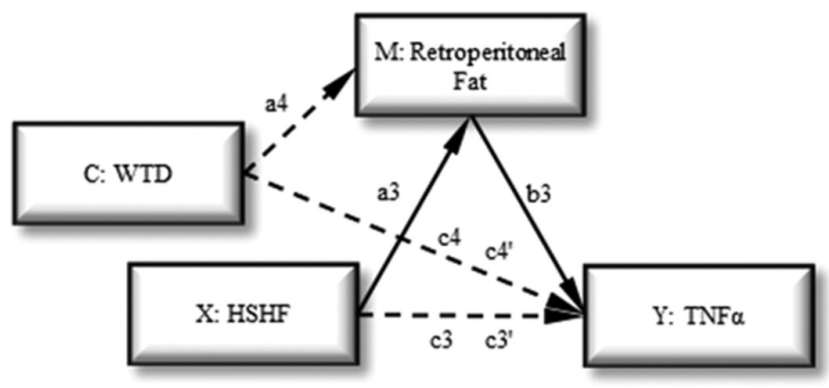

\section{Figure 6}

Mediation models for HSHF intake effect on hepatic MyD88 and TNF $\alpha$; (A) model for epididymal fat mediation of intake effect on MyD88; (B) model for retroperitoneal fat mediation of intake effect on TNF $\alpha$; (a) effect of predictor $(X)$ on mediator $(M)$; (b) effect of mediator $(M)$ on outcome $(Y)$ controlling for predictor $(\mathrm{X})$; $\left(\mathrm{C}^{\prime}\right)$ direct effect of predictor $(\mathrm{X})$ on outcome (Y); (C) confounder; (continuous arrow) significant effect; (dashed arrow) non-significant effect.

(Kershaw \& Flier 2004). A higher influx of fatty acids to this tissue, mainly from the diet sources, can promote enlargement of adipocytes, impairing its functions. The excessive size of adipocytes hardens the blood flow by restricting blood vessels diameters, leading to hypoxia. In low oxygen states, there is a rise in pro-inflammatory adipokines, as IL6, IL1 $\beta$, and TNF $\alpha$, besides macrophage migration and polarisation into M1 (Kihira et al. 2014, Lin \& Yun 2015).

Nevertheless, withdrawal promoted lower hepatic IL6 and IL1 $\beta$ levels independently from VAT.
This decline suggests that WTD effect can also be linked to the absence of the negative stimuli from HSHF intake, possibly indicating a role for gut microbiota, which is being consolidated as a critical regulator for inflammatory diseases, endotoxemia, food intake, and obesity (Xiao \& Zhao 2014, Guerville et al. 2017, van de Wouw et al. 2017, Mulders et al. 2018). At the same time, the mesenteric fat - mediator of HSHF intake's effect on p-p65 and IL1 $\beta$ - may be a direct target for inflammatory molecules passing intestinal barrier (e.g. LPS) in an obesogenic feeding condition (Konrad $\&$ Wueest 2014).

Altogether, our study reinforces the relationship between visceral adipose tissue and hepatic health status and postulates that an increase in hepatic inflammation may occur before and independently from hepatic steatosis. Our results showed that hepatic inflammation can be induced by unhealthy eating and can precede significant fat accumulation. Taking into account that the nutrients consumed have inflammatory potential and, that posterior to gut absorption, they enter the liver via the portal circulation, it is highly unlikely that hepatic fat accumulation and inflammation is a one-way road. The elevated levels of pro-inflammatory markers reported here indicate a response from the tissue, although, as seen post-withdrawal, not an irreversible one. The activation of the inflammatory pathways occurs despite the magnitude of the aggression. Although, a consistent and long-term activation with no resolution would promote damage and perhaps histopathological features.

Overall, a 48-h withdrawal was not entirely effective in decreasing hepatic inflammation and also increased non-HDL cholesterol. Since dietary improvement is the recommended nutritional approach to improve health status in an obesogenic-diet-consuming population and little information regarding the dietary modification per se is available, we see this as a promising research field craving for information.

Table 7 Mediation model.

\begin{tabular}{|c|c|c|}
\hline & $\mathbf{a} \boldsymbol{\beta}(\mathrm{z})$ & b $\boldsymbol{\beta}(\mathrm{z})$ \\
\hline $\begin{array}{l}\text { (1) HSHF } \rightarrow \text { Epidydimal Fat } \rightarrow \text { MyD88 } \\
\text { (2) WTD } \rightarrow \text { Epidydimal Fat } \rightarrow \text { MyD88 } \\
\text { (3) HSHF } \rightarrow \text { Retroperitoneal Fat } \rightarrow \text { TNF } \alpha \\
\text { (4) WTD } \rightarrow \text { Retroperitoneal Fat } \rightarrow \text { TNF } \alpha\end{array}$ & $\begin{array}{r}0.76(4.60)^{\mathrm{a}} \\
-0.05(-0.32) \\
0.85(5.23)^{\mathrm{a}} \\
-0.11(-0.70)\end{array}$ & $\begin{array}{l}0.53(2.62)^{\mathrm{a}} \\
0.59(2.28)^{\mathrm{a}}\end{array}$ \\
\hline
\end{tabular}

\begin{tabular}{c}
\hline $\mathbf{c}^{\prime} \boldsymbol{\beta}(\mathrm{z})$ \\
\hline $0.19(0.80)$ \\
$0.04(0.23)$ \\
$0.11(0.39)$ \\
$-0.03(-0.13)$ \\
\hline
\end{tabular}

\begin{tabular}{c}
$\begin{array}{c}\text { Mediated effect } \\
(\mathrm{a} \times \mathrm{b}) \boldsymbol{\beta}(\mathrm{z})\end{array}$ \\
\hline $0.41(2.28)^{\mathrm{a}}$ \\
$-0.03(-0.32)$ \\
$0.50(2.09)^{\mathrm{a}}$ \\
$-0.07(-0.67)$ \\
\hline
\end{tabular}

Total effect $c$ $\left(a \times b+c^{\prime}\right) \boldsymbol{\beta}(z)$ $0.59(3.06)^{a}$

$0.01(0.06)$

$0.62(2.86)^{\mathrm{a}}$ $-0.09(-0.43)$

Effect size (standardised $\beta$ ), $z$ value, and significance of the models' coefficients.

a indicate $P<0.05$.

$\rightarrow$, orientation of effect; a, effect of predictor $(X)$ on mediator $(M)$; b, effect of mediator $(M)$ on outcome $(Y)$ controlling for predictor $(X)$; $C^{\prime}$, direct effect of predictor (X) on outcome (Y); HSHF, high-sugar and high fat diet; MyD88, myeloid differentiation factor 88; WTD, withdrawal. 


\section{Declaration of interest}

The authors declare that there is no conflict of interest that could be perceived as prejudicing the impartiality of the research reported.

\section{Funding}

This work was supported in part by the 'Coordination for the Improvement of Higher Education Personnel' (CAPES Brazil - Financial Code 001) and by 'São Paulo Research Foundation' (FAPESP \#2017/25420-3).

\section{References}

Aguiar O, Gollücke AP, de Moraes BB, Pasquini G, Catharino RR, Riccio MF, Ihara SS \& Ribeiro DA 2011 Grape juice concentrate prevents oxidative DNA damage in peripheral blood cells of rats subjected to a high-cholesterol diet. British Journal of Nutrition $\mathbf{1 0 5}$ 694-702. (https://doi.org/10.1017/S0007114510004368)

Asrih M \& Jornayvaz FR 2013 Inflammation as a potential link between nonalcoholic fatty liver disease and insulin resistance. Journal of Endocrinology 218 R25-R36. (https://doi.org/10.1530/JOE-13-0201)

Barchetta I, Cimini FA, Ciccarelli G, Baroni MG \& Cavallo MG 2019 Sick fat: the good and the bad of old and new circulating markers of adipose tissue inflammation. Journal of Endocrinological Investigation 42 1257-1272. (https://doi.org/10.1007/s40618-019-01052-3)

Berk PD \& Verna EC 2016 Nonalcoholic fatty liver disease. Lipids and insulin resistance. Clinics in Liver Disease 20 245-262. (https://doi. org/10.1016/j.cld.2015.10.007)

Bortolin RC, Vargas AR, Gasparotto J, Chaves PR, Schnorr CE, Martinello KB, Silveira AK, Rabelo TK, Gelain DP \& Moreira JCF 2018 A new animal diet based on human Western diet is a robust dietinduced obesity model: comparison to high-fat and cafeteria diets in term of metabolic and gut microbiota disruption. International Journal of Obesity 42 525-534. (https://doi.org/10.1038/ijo.2017.225)

Brunner FJ, Waldeyer C, Ojeda F, Salomaa V, Kee F, Sans S, Thorand B, Giampaoli S, Brambilla P, Tunstall-Pedoe H, et al. 2019 Application of non-HDL cholesterol for population-based cardiovascular risk stratification: results from the Multinational Cardiovascular Risk Consortium. Lancet 394 2173-2183. (https://doi.org/10.1016/S01406736(19)32519-X)

Casagrande BP, Gomes MFP, Moura EOC, Santos ACC, Kubota MC, Ribeiro DA, Pisani LP, Medeiros A \& Estadella D 2019 Age-dependent hepatic alterations induced by a high-fat high-fructose diet. Inflammation Research 68 359-368. (https://doi.org/10.1007/s00011-019-01223-1)

Chiba T, Noji K, Shinozaki S, Suzuki S, Umegaki K \& Shimokado K 2016 Diet-induced non-alcoholic fatty liver disease affects expression of major cytochrome P450 genes in a mouse model. Journal of Pharmacy and Pharmacology 68 1567-1576. (https://doi.org/10.1111/jphp.12646)

Cornide-Petronio ME, Jiménez-Castro MB, Gracia-Sancho J \& Peralta C 2019 New insights into the liver-visceral adipose axis during hepatic resection and liver transplantation. Cells 8 1100. (https://doi. org/10.3390/cells8091100)

Das N, Mandala A, Bhattacharjee S, Mukherjee D, Bandyopadhyay D \& Roy SS 2017 Dietary fat proportionately enhances oxidative stress and glucose intolerance followed by impaired expression of the genes associated with mitochondrial biogenesis. Food and Function $\mathbf{8}$ 1577-1586. (https://doi.org/10.1039/C6FO01326K)

de Vos AF, van Haren MA, Verhagen C, Hoekzema R \& Kijlstra A 1994 Kinetics of intraocular tumor necrosis factor and interleukin- 6 in endotoxin-induced uveitis in the rat. Investigative Ophthalmology and Visual Science 35 1100-1106.

Feghali CA \& Wright TM 1997 Cytokines in acute and chronic inflammation. Frontiers in Bioscience 2 d12-d26. (https://doi.org/10.2741/a171)

Folch J, Lees M \& Sloane Stanley GH 1957 A simple method for the isolation and purification of total lipides from animal tissues. Journal of Biological Chemistry 226 497-509.
Franchitto A, Carpino G, Alisi A, De Peppo F, Overi D, De Stefanis C, Romito I, De Vito R, Caccamo R, Sonia B, et al. 2019 The contribution of the adipose tissue-liver axis in pediatric patients with nonalcoholic fatty liver disease after laparoscopic sleeve gastrectomy. Journal of Pediatrics 216 117.e2-127.e2. (https://doi.org/10.1016/j. jpeds.2019.07.037)

Guerville M, Leroy A, Sinquin A, Laugerette F, Michalski MC \& Boudry G 2017 Western-diet consumption induces alteration of barrier function mechanisms in the ileum that correlates with metabolic endotoxemia in rats. American Journal of Physiology: Endocrinology and Metabolism 313 E107-E120. (https://doi.org/10.1152/ajpendo.00372.2016)

Hazarika A, Kalita H, Chandra Boruah D, Chandra Kalita M \& Devi R 2016 Pathophysiology of metabolic syndrome: the onset of natural recovery on withdrawal of a high-carbohydrate, high-fat diet. Nutrition 32 1081-1091. (https://doi.org/10.1016/j.nut.2016.03.005)

Ishtiaq SM, Rashid H, Hussain Z, Arshad MI \& Khan JA 2019 Adiponectin and PPAR: a setup for intricate crosstalk between obesity and non-alcoholic fatty liver disease. Reviews in Endocrine and Metabolic Disorders 20 253-261. (https://doi.org/10.1007/s11154-019-09510-2)

Jeffery RW, Wing RR \& French SA 1992 Weight cycling and cardiovascular risk factors in obese men and women. American Journal of Clinical Nutrition 55 641-644. (https://doi.org/10.1093/ajcn/55.3.641)

Jensen MD, Ryan DH, Apovian CM, Ard JD, Comuzzie AG, Donato KA, Hu FB, Hubbard VS, Jakicic JM, Kushner RF, et al. 2014 Reprint: 2013 AHA/ACC/TOS Guideline for the management of overweight and obesity in adults. Journal of the American Pharmacists Association 2013 54. (https://doi.org/10.1331/JAPhA.2014.14502)

Jin Y \& Fu J 2019 Novel insights Into the NLRP3 inflammasome in atherosclerosis. Journal of the American Heart Association 8 e012219. (https://doi.org/10.1161/JAHA.119.012219)

Kabat AM \& Pearce EJ 2017 Inflammation by way of macrophage metabolism. Science 356 488-489. (https://doi.org/10.1126/science. aan2691)

Kanwar P \& Kowdley KV 2016 The metabolic syndrome and its influence on nonalcoholic steatohepatitis. Clinics in Liver Disease 20 225-243. (https://doi.org/10.1016/j.cld.2015.10.002)

Kershaw EE \& Flier JS 2004 Adipose tissue as an endocrine organ. Journal of Clinical Endocrinology and Metabolism 89 2548-2556. (https://doi org/10.1210/jc.2004-0395)

Kihira Y, Miyake M, Hirata M, Hoshina Y, Kato K, Shirakawa H, Sakaue H, Yamano N, Izawa-Ishizawa Y, Ishizawa K, et al. 2014 Deletion of hypoxia-inducible factor- $1 \alpha$ in adipocytes enhances glucagon-like peptide- 1 secretion and reduces adipose tissue inflammation. PLoS ONE 9 e93856. (https://doi.org/10.1371/journal.pone.0093856)

Kilkenny C, Browne WJ, Cuthill IC, Emerson M \& Altman DG 2010 Improving bioscience research reporting: the arrive guidelines for reporting animal research. PLoS Biology 8 e1000412. (https://doi. org/10.1371/journal.pbio.1000412)

Konrad D \& Wueest S 2014 The gut-adipose-liver axis in the metabolic syndrome. Physiology 29 304-313. (https://doi.org/10.1152/ physiol.00014.2014)

Konstantynowicz-Nowicka K, Berk K, Chabowski A, Kasacka I, Bielawiec P, Łukaszuk B \& Harasim-Symbor E 2019 High-fat feeding in time-dependent manner affects metabolic routes leading to nervonic acid synthesis in NAFLD. International Journal of Molecular Sciences 20 3829. (https://doi.org/10.3390/ijms20153829)

Lin Q \& Yun Z 2015 The hypoxia-inducible factor pathway in adipocytes: the role of HIF-2 in adipose inflammation and hypertrophic cardiomyopathy. Frontiers in Endocrinology 6 39. (https://doi. org/10.3389/fendo.2015.00039)

Liu L, Wang S, Yao L, Li JX, Ma P, Jiang LR, Ke DZ, Pan YQ \& Wang JW $2016 a$ Long-term fructose consumption prolongs hepatic stearoylCoA desaturase 1 activity independent of upstream regulation in rats. Biochemical and Biophysical Research Communications 479 643-648. (https://doi.org/10.1016/j.bbrc.2016.09.160)

Liu J, Han L, Zhu L \& Yu Y 2016b Free fatty acids, not triglycerides, are associated with non-alcoholic liver injury progression in high fat diet 
induced obese rats. Lipids in Health and Disease 15 27. (https://doi. org/10.1186/s12944-016-0194-7)

Liu T, Zhang L, Joo D \& Sun SC 2017 NF-kB signaling in inflammation. Signal Transduction and Targeted Therapy 2 17023. (https://doi. org/10.1038/sigtrans.2017.23)

Lozano I, Van der Werf R, Bietiger W, Seyfritz E, Peronet C, Pinget M, Jeandidier N, Maillard E, Marchioni E, Sigrist S, et al. 2016 Highfructose and high-fat diet-induced disorders in rats: impact on diabetes risk, hepatic and vascular complications. Nutrition and Metabolism 13 15. (https://doi.org/10.1186/s12986-016-0074-1)

Lu YC, Yeh WC \& Ohashi PS 2008 LPS/TLR4 signal transduction pathway. Cytokine 42 145-151. (https://doi.org/10.1016/j. cyto.2008.01.006)

Martire SI, Maniam J, South T, Holmes N, Westbrook RF \& Morris M] 2014 Extended exposure to a palatable cafeteria diet alters gene expression in brain regions implicated in reward, and withdrawal from this diet alters gene expression in brain regions associated with stress. Behavioural Brain Research 265 132-141. (https://doi. org/10.1016/j.bbr.2014.02.027)

Montoya AK \& Hayes AF 2017 Two-condition within-participant statistical mediation analysis: a path-analytic framework. Psychological Methods 22 6-27. (https://doi.org/10.1037/met0000086)

Morris MJ, Beilharz JE, Maniam J, Reichelt AC \& Westbrook RF 2015 Why is obesity such a problem in the 21st century? The intersection of palatable food, cues and reward pathways, stress, and cognition. Neuroscience and Biobehavioral Reviews 58 36-45. (https://doi. org/10.1016/j.neubiorev.2014.12.002)

Mulders RJ, de Git KCG, Schéle E, Dickson SL, Sanz Y \& Adan RAH 2018 Microbiota in obesity: interactions with enteroendocrine, immune and central nervous systems. Obesity Reviews 19 435-451. (https://doi. org/10.1111/obr.12661)

Musso G, Gambino R, Durazzo M, Biroli G, Carello M, Fagà E, Pacini G, De Michieli F, Rabbione L, Premoli A, et al. 2005 Adipokines in NASH: postprandial lipid metabolism as a link between adiponectin and liver disease. Hepatology 42 1175-1183. (https://doi.org/10.1002/hep.20896)

Rinnankoski-Tuikka R, Hulmi JJ, Torvinen S, Silvennoinen M, Lehti M, Kivelä R, Reunanen H, Kujala UM \& Kainulainen H 2014 Lipid droplet-associated proteins in high-fat fed mice with the effects of voluntary running and diet change. Metabolism: Clinical and Experimental 63 1031-1040. (https://doi.org/10.1016/j. metabol.2014.05.010)

Rocha DM, Caldas AP, Oliveira LL, Bressan J \& Hermsdorff HH 2016 Saturated fatty acids trigger TLR4-mediated in flammatory response. Atherosclerosis 244 211-215. (https://doi.org/10.1016/j. atherosclerosis.2015.11.015)

Santamarina AB, Jamar G, Mennitti LV, de Rosso VV, Cesar HC, Oyama LM \& Pisani LP 2018 The use of Juçara (Euterpe edulis mart.) supplementation for suppression of NF-кB pathway in the hypothalamus after high-fat diet in Wistar rats. Molecules 231814. (https://doi.org/10.3390/molecules23071814)

Santamarina AB, Jamar G, Mennitti LV, Ribeiro DA, Cardoso CM, De Rosso VV, Oyama LM \& Pisani LP 2019 Polyphenols-rich fruit (Euterpe edulis mart.) prevents peripheral inflammatory pathway activation by the short-term high-fat diet. Molecules 24 1-14. (https:// doi.org/10.3390/molecules24091655)

Shang Y, Khafipour E, Derakhshani H, Sarna LK, Woo CW, Siow YL \& O K 2017 Short term high fat diet induces obesity-enhancing changes in mouse gut microbiota that are partially reversed by cessation of the high fat diet. Lipids 52 499-511. (https://doi.org/10.1007/s11745-017-4253-2)
Sharma RJ, Macallan DC, Sedgwick P, Remick DG \& Griffin GE 1992 Kinetics of endotoxin-induced acute-phase protein gene expression and its modulation by TNF-alpha monoclonal antibody. American Journal of Physiology 262 R786-R793. (https://doi.org/10.1152/ ajpregu.1992.262.5.R786)

Smith B \& George J 2009 Adipocyte-hepatocyte crosstalk and the pathogenesis of nonalcoholic fatty liver disease. Hepatology $\mathbf{4 9}$ 1765-1767. (https://doi.org/10.1002/hep.22937)

Totsch SK, Quinn TL, Strath LJ, McMeekin LJ, Cowell RM, Gower BA \& Sorge RE 2017 The impact of the Standard American Diet in rats: effects on behavior, physiology and recovery from inflammatory injury. Scandinavian Journal of Pain 17 316-324. (https://doi. org/10.1016/j.sjpain.2017.08.009)

Ullman JB \& Bentler PM 2017 Structural equation modeling. In Handbook of Psychology, 2nd ed., pp. 557-580. Ed IB Weiner. Hoboken, NJ, USA: John Wiley \& Sons. (https://doi.org/10.1007/978-1-4939-7274-6_28)

Unamuno X, Gómez-Ambrosi J, Ramírez B, Rodríguez A, Becerril S, Valentí V, Moncada R, Silva C, Salvador J, Frühbeck G, et al. 2019 NLRP3 inflammasome blockade reduces adipose tissue inflammation and extracellular matrix remodeling. Cellular and Molecular Immunology [epub]. (https://doi.org/10.1038/s41423-019-0296-z)

van de Wouw M, Schellekens H, Dinan TG \& Cryan JF 2017 Microbiotagut-brain axis: modulator of host metabolism and appetite. Journal of Nutrition 147 727-745. (https://doi.org/10.3945/jn.116.240481)

Van Herck MA, Vonghia L \& Francque SM 2017 Animal models of nonalcoholic fatty liver disease - a starter's guide. Nutrients 91072. (https://doi.org/10.3390/nu9101072)

Wagnerberger S, Spruss A, Kanuri G, Volynets V, Stahl C, Bischoff SC \& Bergheim I 2012 Toll-like receptors 1-9 are elevated in livers with fructose-induced hepatic steatosis. British Journal of Nutrition 107 1727-1738. (https://doi.org/10.1017/S0007114511004983)

Xiao S \& Zhao L 2014 Gut microbiota-based translational biomarkers to prevent metabolic syndrome via nutritional modulation. FEMS Microbiology Ecology 87 303-314. (https://doi.org/10.1111/15746941.12250)

Zafar U, Khaliq S, Ahmad HU, Manzoor S \& Lone KP 2018 Metabolic syndrome: an update on diagnostic criteria, pathogenesis, and genetic links. Hormones 17 299-313. (https://doi.org/10.1007/s42000-0180051-3)

Zamarron BF, Mergian TA, Cho KW, Martinez-Santibanez G, Luan D, Singer K, DelProposto JL, Geletka LM, Muir LA \& Lumeng CN 2017 Macrophage proliferation sustains adipose tissue inflammation in formerly obese mice. Diabetes 66 392-406. (https://doi.org/10.2337/ db16-0500)

Zeigler ZS, Birchfield N, Moreno K, James D \& Swan P 2018 Fatness and fluctuating body weight: effect on central vasculature. BioResearch Open Access 7 90-100. (https://doi.org/10.1089/ biores.2017.0044)

Zhang Y, Proenca R, Maffei M, Barone M, Leopold L \& Friedman JM 1994 Positional cloning of the mouse obese gene and its human homologue. Nature 372 425-432. (https://doi.org/10.1038/372425a0)

Zhang J, Zhao Y, Xu C, Hong Y, Lu H, Wu J \& Chen Y 2014 Association between serum free fatty acid levels and nonalcoholic fatty liver disease: a cross-sectional study. Scientific Reports 4 5832. (https://doi. org/10.1038/srep05832)

Zou H, Yin P, Liu L, Liu W, Zhang Z, Yang Y, Li W, Zong Q \& Yu X 2019 Body-weight fluctuation was associated with increased risk for cardiovascular disease, all-cause and cardiovascular mortality: a systematic review and meta-analysis. Frontiers in Endocrinology 10728. (https://doi.org/10.3389/fendo.2019.00728)

Received in final form 11 March 2020

Accepted 25 March 2020

Accepted Manuscript published online 25 March 2020 https://joe.bioscientifica.com https://doi.org/10.1530/JOE-20-0073
(C) 2020 Society for Endocrinology Published by Bioscientifica Ltd. Printed in Great Britain 